\title{
Adenoid Hypertrophy in Adults: An Overlooked Diagnosis
}

\section{Pooja Das, Vikas Kakkar, Sahil Kapoor, Poonam Kumar Saidha* and Mithun Abraham}

Department of ENT, SGT University, India

*Corresponding Author: Poonam Kumar Saidha, Associate Professor, Department of ENT, SGT University, India.
Received: November 08, 2021

Published: November 29, 2021

(C) All rights are reserved by Poonam Kumar

Saidha., et al.

\section{Abstract}

Aim: Adenoid hypertrophy is a common finding in children. Adenoids are present since birth, show physiological hypertrophy in children between the age 6-10 years and atrophies at adolescence. Although its finding in adults causing nasal obstruction is uncommon but the incidence has been increasing these days.

Methods: This is a retrospective study of 60 patients with complaints of nasal obstruction, in which 50 patients were above 18 years and remaining 10 patients were in the paediatric age group. These 50 adult patients were selected for the study and examined thoroughly. All routine investigations were sent including imaging modalities like X ray nasopharynx and Diagnostic nasal endoscopy which revealed the presence of adenoid tissue in these selected adult patients. Microbiological examination of throat swab, eosinophil count, serum IgE, absolute eosinophil count was conducted to exclude other cause of nasal blockage like acute rhinitis, rhino sinusitis or allergic rhinitis. Patients were posted for adenoidectomy.

Results: Postoperative event remained uneventful, histopathology report revealed normal adenoid tissue of lymphoid origin. Adenoid hypertrophy was seen in adults who were exposed to pollution and were a habitual smoker.

Conclusion: Presence of adenoid tissue is a common finding in children but its incidence in adults are observed due to increased rate of infection, allergy and malignancy. Smoking and pollution is considered to be a predisposing factor. Henceforth adenoid hypertrophy should be considered in adult patients complaining of nasal obstruction as it can be a causative factor or indication of chronic inflammation for which early diagnosis and management must be contemplated.

Keywords: Adenoid Hypertrophy; Adult Nasal Obstruction; Nasal Endoscopy; X-ray Nasopharynx; Pollution

\section{Introduction}

Adenoid is an aggregation of lymphoid tissue located at the posterosuperior wall of nasopharynx [1]. The adenoid forms part of Waldeyer's ring of lymphoid tissue at the portal of the upper respiratory tract. They act as the first site of immunological contact for inhaled antigens encountered in childhood. Adenoid produces B cells which further give rise to IgG, IgA and plasma cells, hence they appear to have an important role in the development of Im- munological Memory in younger children [2]. Adenoids are present at birth, shows physiologically hypertrophy in children between the age of 6-10 years, then atrophies at adolescence [3]. Clinical symptoms are more common in children due to small volume of nasopharynx and repeated attacks of upper respiratory tract infections. Adenoid hypertrophy was considered uncommon in adults as examination of nasopharynx by posterior rhinoscopy was inadequate, hence many cases of nasal obstruction in adults due to 
adenoids were misdiagnosed and incompletely treated [4]. However, in current clinical practice with advent of nasal endoscopies and improved imaging modalities, detection of adenoidal tissue is encountered routinely. Inspite of all these developments there is a scarcity in the data of incidence regarding adult adenoid hypertrophy.

Present study is a retrospective case series of adult patients who underwent adenoidectomy with history of nasal obstruction

\section{Material and Methods}

- $\quad$ Study Design - Retrospective study of 60 patients who underwent adenoidectomy with history of nasal obstruction, from which 50 patients were above 18 years and remaining 10 patients were below 18 years.

- $\quad$ Study Setting - This is a hospital based study conducted in the Department of ENT at a tertiary care hospital

- $\quad$ Study Period - March 2017- September 2019

- $\quad$ This study titled - "Adenoid Hypertrophy in Adults: An Overlooked Diagnosis". is approved by Institutional Research committee and Institutional Ethics committee our University.

Inclusion criteria

- $\quad$ Age of patient above 18 years

- Patient complaining of nasal obstruction

- Patient having enlarged adenoids on Diagnostic Nasal Endoscopy and on X-Ray.

Exclusion criteria

- $\quad$ Age of patient below 06 years

- $\quad$ Patient diagnosed with any acute infection like acute tonsillitis, acute rhinitis or sinusitis or any acute upper respiratory tract infections

- $\quad$ Patients diagnosed with Allergic rhinitis.

\section{Observation}

Approach for the study was designed as such that 60 patients complaining of nasal obstruction were randomly selected, in which 50 patients belonged to adult age group and the remaining 10 patients were in the pediatric age group. These 50 patients were evaluated and studied.

History of these 50 patients were taken thoroughly and other related symptoms like sneezing, rhinorrhea, itching of nose, headache, fever, loss of smell, cough etc. were also taken to exclude additional causes of nasal obstruction like DNS, allergic rhinitis, rhino-sinusitis. Admissible past history and family history was also taken into consideration followed by detailed clinical examination including both general and local examination. Anterior rhinoscopy was useful to detect deviated nasal septum, turbinate hypertrophy, or nasal polyp. Posterior rhinoscopy was not much informative regarding adenoid hypertrophy as digital palpation of adenoid tissues is not possible in mirror examination. To confirm the findings of posterior rhinoscopy, diagnostic nasal endoscopy was done where adenoids were visualized in the posterior choanae (as shown in figure 2). All basic routine investigations were done. Imaging modalities like X-ray PNS Water's view, X-ray nasopharynx lateral view with extended neck and open mouth was done to visualize the shadow of adenoid (as illustrated in figure 1).

The degree of nasopharyngeal obstruction was determined by assessing the ratio of adenoid shadow diameter to nasopharyngeal diameter.

- $\quad$ Mild- $<50 \%$ of palatal airway obstructed

- $\quad$ Moderate- $>50 \%$ obstruction of palatal airway

- $\quad$ Severe- complete nasopharyngeal obstruction with no air column seen on the post nasal space [5].

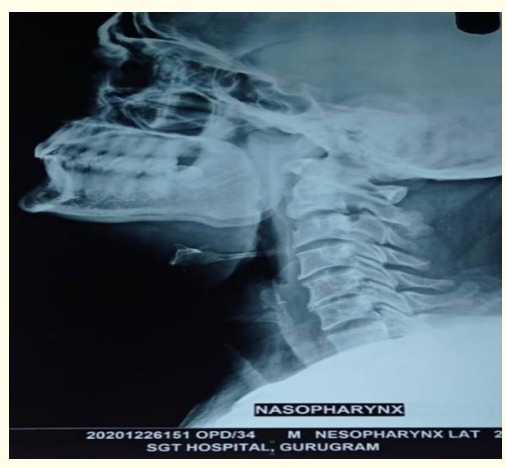

Figure 1: X-ray Nasopharynx Lateral view showing adenoid shadow. 
Nasal endoscopic grading of adenoids

- Grade 1- Adenoid tissue filling one third of vertical portion of choanae

- Grade 2- Adenoid tissue filling one third to two third of the choanae

- $\quad$ Grade 3- From two third to nearly complete obstruction of the choanae

- $\quad$ Grade 4- Complete choanal obstruction [2].

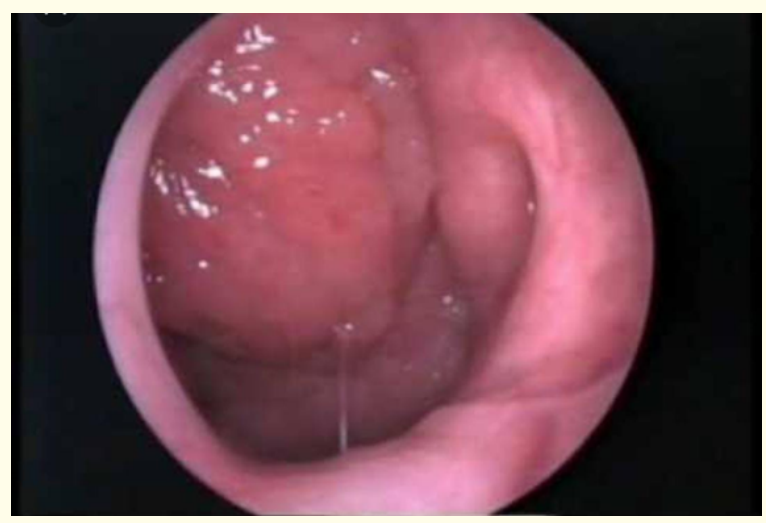

Figure 2: Nasal endoscopy showing adenoid tissue in roof of nasopharynx.

Throat swab was sent for culture and sensitivity which revealed no growth of any organism. Hematological investigation like differential leucocyte count, absolute eosinophil count, nasal smear for eosinophil and serum Ig E was taken to rule out allergic cause of nasal obstruction. After confirmation of adenoid mass, these patients were posted for adenoidectomy under general anesthesia and the adenoid tissue was sent for histopathological examination. Post-operative period went uneventful and patient responded well with subsequent relief in the nasal obstruction. Histopathological report yielded normal adenoid tissue of lymphoid origin.

\section{Results}

In this study out of 60 patients, 36 patients were male and 24 patients were female from which 50 were in adult group and remaining 10 were in the pediatric age group. Common pathology in pediatric age were chronic tonsillitis with adenoid hypertrophy whereas in adult group pathology was solely adenoid hypertrophy without any other nasal pathology or component of allergy (as shown in table 1). Hence prevalence of adenoid hypertrophy in adults from this study results to be $83.32 \%$.

\begin{tabular}{|c|c|c|c|c|c|}
\hline $\begin{array}{c}\text { Serial } \\
\text { no }\end{array}$ & Pathology & $\begin{array}{c}\text { Age in } \\
\text { years }\end{array}$ & Male & Female & Percentage \\
\hline 1 & Adenoid hy- & 22 & 14 & 09 & 38.33 \\
& pertrophy & 28 & 09 & 05 & 23.33 \\
& & 35 & 08 & 05 & 21.66 \\
\hline 2 & Chronic ton- & 8 & 02 & 03 & 8.33 \\
& sillitis with & 12 & 03 & 02 & 8.33 \\
& adenoid & & & & \\
\hline 3 & hypertrophy & & & & 99.99 \\
\hline
\end{tabular}

Table 1: Causes of nasal obstruction $(n=60)$.

From this tabulation total 50 adult patients were found to have adenoid hypertrophy and the prevalence of adenoid hypertrophy in adults causing nasal obstruction $83.32 \%$.

Among these 50 adult patients, 31 were male and 19 were female patients. It shows that males are more commonly involved than female. As per habitat distribution 39 were from rural area and only 11 patients were from urban area. On observing the occupation and personal habits of the rural patients it was found that the possible predisposing factor for male preponderance of adenoid hypertrophy is due to their working environment in agricultural fields thus exposing them to pollution and an added factor of hookah smoking which is more prevalent in the rural belt of Haryana (as shown in table 2). This implies that pollution and smoking may be an important factor in the development of adenoid hypertrophy along with chronic infection.

\begin{tabular}{|c|c|c|c|}
\hline $\begin{array}{c}\text { Serial } \\
\text { no }\end{array}$ & $\begin{array}{c}\text { Precipitating } \\
\text { factors }\end{array}$ & $\begin{array}{c}\text { No of patients } \\
\text { from rural area }\end{array}$ & $\begin{array}{c}\text { No of patients } \\
\text { from urban area }\end{array}$ \\
\hline 1 & Pollution & 13 & 07 \\
\hline 2 & Smoking & 26 & 04 \\
\hline 3 & Total & 39 & $11(39+11=50)$ \\
\hline
\end{tabular}

Table 2: Predisposing factors for adult adenoid hypertrophy $(\mathrm{n}=50)$.

\section{Discussion}

Adenoids are the condensation of lymphoid tissue situated on the posterior-superior wall of nasopharynx [1]. The adenoid tis- 
sue along with the tonsils form the first line of defense mechanism in the childhood as it prevents the entry of pathogenic organisms through nose and mouth. They are largely composed of B lymphocytes which plays role of making antibodies on contact with any pathogen [2]. Adenoids like tonsillar tissue can also be involved with both acute and chronic infections. With ongoing infection or inflammation, the adenoids can progressively enlarge. As adenoids are located posteriorly in the nasopharynx its hypertrophy affects nasal function [6]. Adenoids play an important role for immunological memory in younger children. Adenoids are present since birth, show physiological hypertrophy at 6-10 years and atrophies at adolescence [3]. Although adenoid regression is seen towards the adolescent period but hypertrophy of adenoid tissue is also observed in adult population [7]. Presence of lymphoid hyperplasia in adult nasopharynx including persistence of childhood adenoids is associated with chronic inflammation [4].

Regressed adenoidal tissue may re-proliferate in response to infection and irritants [8]. There are various clinical features associated with adenoid hypertrophy. Patients complaint of nasal obstruction which results in oral breathing, recurrent nasal infection and hypo-nasal speech $[4,8]$.

Long term adenoidal enlargement can lead to ear discharge or ear pain due to obstruction to pharyngeal opening of Eustachian tube and chronic mouth breathing. Prolonged mouth breathing can further cause orthodontic abnormalities or dento-skeletal malocclusion [6]. Besides allergy and chronic infection, smoking is also believed to be a predisposing factor for adenoidal hyperplasia. Finkelstein., et al. reported the presence of obstructive adenoids in $30 \%$ of smokers [9].

On detection of presence of adenoid tissue, malignancies of type B white blood cell (lymphoma plasmacytoma) or HIV must be considered. Normally in acute condition, adenoids respond to antibiotics and steroid nasal sprays and regress to a smaller size providing relief from nasal complaints, but if it re-enlarges or in refractory cases surgery becomes the line of treatment [6]. Adult adenoid hypertrophy can be an alarming state as it can be associated with lymphoma or other malignancy and risk of HIV infection cannot be spared, hence early diagnosis and management must be contemplated.

\section{Clinical significance}

Nasal obstruction is one of the commonest complaint related to nose. Complaint of nasal obstruction encountered in adult population is mostly seen due to DNS, turbinate hypertrophy, allergic rhinitis and other nasal mass. Adenoid hypertrophy resulting in nasal obstruction in adults is often over looked and hence its diagnosis is missed and undertreated. Hence forth adenoid hypertrophy must be kept in mind even though it might not be the commonest cause encountered in adults.

\section{Conclusion}

Presence of adenoid hypertrophy as a cause for nasal obstruction is a common finding noticed in children, although its presence in adults is considered uncommon. But now the incidence of adenoid hypertrophy in adults is increasing due to allergy, chronic infection and malignancy. Pollution is observed to be a predisposing factor. This study shows that $83.32 \%$ of adult nasal obstruction amongst total of 60 selected patients is due to adenoid hypertrophy. Males are more commonly affected than female due to outdoor activities and exposure of pollutants. Association of malignant sino-nasal tumors, lymphomas and HIV infections are rare but should be kept in survey as treatment at an initial stage can be commenced. Decisively, adenoid hypertrophy must be scrutinized in adult patients complaining of nasal obstruction as this being an uncommon entity can result to be a causative factor which gets unnoticed and mistreated.

\section{Bibliography}

1. Wysoeka J., et al. "Naïve and memory T cells in hypertrophied adenoids in children according to age". International Journal of Pediatric Otorhinolaryngology 67 (2003): 237-241.

2. Watkinson JC and Clarke RW. The Adenoid and Adenoidectomy (section 1, chapter 26) Scott- Brown's Otorhinolaryngology Head and Neck Surgery, London, Taylor and Francis Group (8th Edition) 2 (2018): 285-87.

3. Yildrim N., et al. "Adenoid hypertrophy in adults: clinical and morphological characteristics". Journal of International Medical Research 36 (2008): 157-162.

4. Kamel RH and Ishak EA. "Enlarged adenoid and adenoidectomy in adults: endoscopic approach and histopathological study". Journal of Laryngology and Otology 104 (1990): 965967. 
5. Bitar MA., et al. "A suggested clinical score to predict the severity of adenoid obstruction in children". European Archives of Oto-Rhino-Laryngology 263 (2006): 924-928.

6. Rout MR., et al. "Adenoid Hypertrophy in Adults: A case series". Indian Journal of Otolaryngology and Head and Neck Surgery 65.3 (2013): 269-274.

7. Minnigerode B and Blass K. "Persistent adenoid hypertrophy". HNO 22 (1974): 347-349.

8. Frenkiel S., et al. "Persistent adenoid hypertrophy presenting as a nasopharyngeal mass". Journal of Otolaryngology 9 (1980): 357-360.

9. Finkelstein Y., et al. "Characterization of smoking induced nasopharyngeal lymphoid hyperplasia”. Laryngoscope 107 (1997): 1635-1642.

Volume 3 Issue 12 December 2021

(C) All rights are reserved by Poonam Kumar Saidha., et al. 